\title{
Erratum to: Birationally rigid hypersurfaces
}

\author{
Tommaso de Fernex ${ }^{1}$
}

Published online: 13 August 2015

(C) Springer-Verlag Berlin Heidelberg 2015

\section{Erratum to: Invent Math (2013) 192:533-566 DOI 10.1007/s00222-012-0417-0}

This note points out a gap in the proof of the main theorem of the article "Birationally rigid hypersurfaces" published in Invent. Math. 192 (2013), 533566 , and provides a new proof of the theorem.

The statements of Lemma 9.1 and Lemma 9.2 in the original article are incorrect (see the remarks at the end of this note for further comments on the errors). The two lemmas are used in the proof of Theorem A, which is the main theorem of the original article; more precisely, they are used in the proof of Theorem 7.4 from which Theorem A is deduced. The error in Lemma 9.2 was brought to our attention by János Kollár.

While Lemma 9.2 is not essential for the proof and can be circumvented, the gap left by the error in Lemma 9.1 appears to be more substantial because of the key role that Lemma 9.1 plays in the application of Theorem B in the proof of Theorem A.

In this note, we give a new argument to prove Theorem A which does not use Theorem B. This does not fix, however, the proof of Theorem 7.4, which should

The online version of the original article can be found under doi:10.1007/s00222-012-0417-0.

Tommaso de Fernex

defernex@math.utah.edu

1 Department of Mathematics, University of Utah, 155 South 1400 East, Salt Lake City, UT 48112-0090, USA 
therefore be considered unproven. We use the same notation and conventions as in the original article.

Theorem (Theorem A of the original article) For $N \geq 4$, every smooth complex hypersurface $X \subset \mathbb{P}^{N}$ of degree $N$ is birationally superrigid.

Proof We assume that $N \geq 7$ and refer to [1] for the remaining cases $4 \leq$ $N \leq 6$.

Suppose that $\phi: X \rightarrow X^{\prime}$ is a birational map, but not an isomorphism, from $X$ to a Mori fiber space $X^{\prime}$. The map is defined by a linear system $\mathcal{H}$ whose members are cut out by homogeneous forms of some degree $r$. Let $D, D^{\prime} \in \mathcal{H}$ be two general elements, and denote

$$
c:=\operatorname{can}(X, D)
$$

Proposition 7.3 of the original article implies that $c<1 / r$. On the other hand, Proposition 8.7 of the original article implies that the set of points $Q \in X$ such that $e_{Q}(D)>r$ is finite. It follows that the pair $(X, c D)$ is terminal in dimension one, and hence there is a closed point $P \in X$ such that $\operatorname{mld}(P ; X, c D)=1$. This implies that $\operatorname{mld}(P ; X, c D+P) \leq 0$.

Let $Y \subset X$ be a general hyperplane section through the point $P$, and let $B:=D \cap D^{\prime} \cap Y$. We remark that $Y$ is a smooth hypersurface of degree $N$ in $\mathbb{P}^{N-1}$ and $B$ is a complete intersection subscheme of $Y$ of codimension two. By inversion of adjunction (e.g., see Theorem 6.1 of the original article), we have $\operatorname{mld}(P ; Y, c B) \leq 0$. This means that $(Y, c B)$ is not log terminal near $P$. Notice, though, that $(Y, c B)$ is $\log$ terminal in dimension one. In fact, we have the following stronger property.

Lemma 1 The pair $(Y, 2 c B)$ is log terminal in dimension one.

Proof Let $C \subset Y$ be any irreducible curve.

Proposition 8.7 of the original article implies that the set of points $Q \in$ $X$ such that $e_{Q}\left(D \cap D^{\prime}\right)>r^{2}$ has dimension at most one. It follows by Proposition 8.5 of the original article that, for a general choice of $Y$, the set of points $Q \in Y$ such that $e_{Q}(B)>r^{2}$ is zero dimensional. Therefore we have $e_{Q}(B) \leq r^{2}$ for a general point $Q \in C$.

Fix such a point $Q \in C$, and let $S \subset Y$ be a smooth surface cut out by general hyperplanes through $Q$. By Proposition 8.5 of the original article, we have $e_{Q}\left(\left.B\right|_{S}\right) \leq r^{2}$. Since $\left.B\right|_{S}$ is a zero-dimensional complete intersection subscheme of $S$, the multiplicity $e_{Q}\left(\left.B\right|_{S}\right)$ is equal to the Hilbert-Samuel multiplicity of the ideal $\mathcal{I}_{\left.B\right|_{S}, Q} \subset \mathcal{O}_{S, Q}$ locally defining $\left.B\right|_{S}$ near $Q$. Then Theorem 0.1 of [2] implies that the $\log$ canonical threshold of $\left(S,\left.B\right|_{S}\right)$ near $Q$ satisfies the inequality 


$$
\operatorname{lct}_{Q}\left(S,\left.B\right|_{S}\right) \geq \frac{2}{\sqrt{e_{Q}(B \mid S)}} .
$$

Since $e_{Q}\left(\left.B\right|_{S}\right) \leq r^{2}$ and $c<1 / r$, this implies that $\operatorname{lct}_{Q}\left(S,\left.B\right|_{S}\right)>2 c$, and hence $\left(S,\left.2 c B\right|_{S}\right)$ is $\log$ terminal near $Q$. It follows by inversion of adjunction that $(Y, 2 c B)$ is $\log$ terminal near $Q$. As $Q$ was chosen to be a general point of an arbitrary curve $C$ on $Y$, we conclude that $(Y, 2 c B)$ is $\log$ terminal in dimension one.

The lemma implies that the multiplier ideal $\mathcal{J}(Y, 2 c B)$ defines a zerodimensional subscheme $\Sigma \subset Y$. We have $H^{1}\left(Y, \mathcal{J}(Y, 2 c B) \otimes \mathcal{O}_{Y}(2)\right)=0$ by Nadel's vanishing theorem, since $\omega_{Y}$ is trivial, $B$ is cut out by forms of degree $r$, and $2 c r<2$. It follows that there is a surjection

$$
H^{0}\left(Y, \mathcal{O}_{Y}(2)\right) \rightarrow H^{0}\left(\Sigma, \mathcal{O}_{\Sigma}(2)\right) \cong H^{0}\left(\Sigma, \mathcal{O}_{\Sigma}\right)
$$

(here $\mathcal{O}_{\Sigma}(2) \cong \mathcal{O}_{\Sigma}$ because $\Sigma$ is zero dimensional), and therefore we have

$$
h^{0}\left(\Sigma, \mathcal{O}_{\Sigma}\right) \leq h^{0}\left(Y, \mathcal{O}_{Y}(2)\right)=\left(\begin{array}{c}
N+1 \\
2
\end{array}\right)
$$

Lemma 2 There exists a prime divisor $E$ over $X$ with center $P$ and log discrepancy

$$
a_{E}(X, c B+P) \leq 0
$$

such that the center of $E$ in the blow-up of $X$ at $P$ has positive dimension.

Proof Recall that $\operatorname{mld}(P ; Y, c B) \leq 0$. We fix a $\log$ resolution $f: Y^{\prime} \rightarrow Y$ of $(Y, B+P)$, and take a general hyperplane section $Z \subset Y$ through $P$. Let $Z^{\prime} \subset Y^{\prime}$ be the proper transform of $Z$. By Bertini's theorem, we can ensure that $Z^{\prime}$ intersects transversally the exceptional locus of $f$ and the induced map $Z^{\prime} \rightarrow Z$ is a $\log$ resolution of $\left(Z,\left.B\right|_{Z}+P\right)$.

We have $\operatorname{mld}\left(P ; Z,\left.c B\right|_{Z}\right) \leq 0$ by inversion of adjunction. This means that there is a prime exceptional divisor $F \subset Z^{\prime}$ with center $P$ in $Y$ and $\log$ discrepancy $a_{F}\left(Z,\left.c B\right|_{Z}\right) \leq 0$. There is a unique prime exceptional divisor $E \subset Y^{\prime}$ such that $F$ is an irreducible component of $\left.E\right|_{Z^{\prime}}$. Note that $\left.E\right|_{Z^{\prime}}$ is reduced. Since $E$ is the only prime divisor of $Y^{\prime}$ that is contained in either supports of the inverse images of $B$ and $P$ and whose restriction to $Z^{\prime}$ contains $F$, we have $\operatorname{val}_{E}(B)=\operatorname{val}_{F}\left(\left.B\right|_{Z}\right)$ and $\operatorname{val}_{E}(P)=\operatorname{val}_{F}(P)$. It follows by adjunction formula that

$$
a_{E}(Y, c B+P)=a_{F}\left(Z,\left.c B\right|_{Z}\right) \leq 0 .
$$


We deduce from the fact that $(Y, c B)$ is $\log$ terminal in dimension one that the center of $E$ in $Y$ is equal to $P$. The fact that $E \cap Z^{\prime} \neq \varnothing$ (for a general hyperplane section $Z \subset Y$ through $P$ ) implies that the center of $E$ on the blow-up of $Y$ at $P$ is positive dimensional.

Let $E$ be as in Lemma 2, and let

$$
\lambda:=\frac{\operatorname{val}_{E}(P)}{c \operatorname{val}_{E}(B)} .
$$

In the next two lemmas, we establish opposite bounds on $\lambda$. The proof of the theorem will result by comparing the two bounds.

Lemma $3 \lambda>\frac{1}{N+1}$.

Proof Let $x, y \in \mathfrak{m}_{Y, P}$ be two general linear combinations of a given regular system of parameters of $Y$ at $P$. Since the center of $E$ on the blow-up of $Y$ at $P$ is positive dimensional, by taking $x, y$ general we can ensure that $\operatorname{val}_{E}(f) \leq \operatorname{deg}(f) \operatorname{val}_{E}(P)$ for any nonzero polynomial $f(x, y)$.

Let $d$ be any positive integer such that

$$
d \operatorname{val}_{E}(P) \leq-a_{E}(Y, 2 c B) .
$$

For every nonzero polynomial $f(x, y)$ of degree $\leq d$, we have val $E(f) \leq$ $-a_{E}(Y, 2 c B)$, and therefore $f \notin \mathcal{J}(Y, 2 c B) \cdot \mathcal{O}_{Y, P}$. This means that if $V \subset$ $\mathcal{O}_{Y, P}$ is the $\mathbb{C}$-vector space spanned by the polynomials in $x, y$ of degree $\leq d$, then the quotient map $\mathcal{O}_{Y, P} \rightarrow \mathcal{O}_{\Sigma, P}$ restricts to a injective map $V \hookrightarrow \mathcal{O}_{\Sigma, P}$, and therefore

$$
h^{0}\left(\Sigma, \mathcal{O}_{\Sigma}\right) \geq \operatorname{dim}_{\mathbb{C}} V=\left(\begin{array}{c}
d+2 \\
2
\end{array}\right)
$$

Comparing this inequality with the upperbound on $h^{0}\left(\Sigma, \mathcal{O}_{\Sigma}\right)$ obtained in (1), we conclude that $N>d$. It follows by our assumption on $d$ that

$$
N \operatorname{val}_{E}(P)>-a_{E}(Y, 2 c B) .
$$

This means that $a_{E}(Y, 2 c B-N P)>0$. Note, on the other hand, that

$$
a_{E}(Y, 2 c B-N P)=a_{E}(Y,(2-(N+1) \lambda) c B+P) .
$$

Since $a_{E}(Y, c B+P) \leq 0$ by Lemma 2 , we conclude that $(N+1) \lambda>1$. 
Lemma $4 \lambda<\frac{\sqrt{N}-2}{\sqrt{N}(N-5)}$.

Proof First, we observe that $(N-5) \lambda \leq 1$. In fact, since $\operatorname{lct}_{P}(Y, P)=N-2$, we have

$$
a_{E}(Y,(N-3) \lambda c B+P)=a_{E}(Y,(N-2) P) \geq 0,
$$

and since $a_{E}(Y, c B+P) \leq 0$ by Lemma 2 , we actually get $(N-3) \lambda \leq 1$.

Let $S \subset Y$ be a surface cut out by $N-4$ general hyperplane sections through $P$. Note that $\left.B\right|_{S}$ is a complete intersection zero-dimensional subscheme of $S$ cut out by two forms of degree $r$. We have

$$
a_{E}(Y,(1-(N-5) \lambda) c B+(N-4) P)=a_{E}(Y, c B+P) \leq 0 .
$$

By our initial remark, the pair in the left hand side is effective. We can therefore apply inversion of adjunction, which gives $\operatorname{mld}(P ; S,(1-(N-$ 5) $\left.\lambda)\left.c B\right|_{S}\right) \leq 0$. This means that

$$
\operatorname{lct}_{P}\left(S,\left.B\right|_{S}\right) \leq(1-(N-5) \lambda) c .
$$

By contrast, by using Theorem 0.1 of [2], Bezout's theorem, and the inequality $c<1 / r$, we get the chain of inequalities

$$
\operatorname{lct}_{P}\left(S,\left.B\right|_{S}\right) \geq \frac{2}{\sqrt{e_{P}\left(\left.B\right|_{S}\right)}} \geq \frac{2}{r \sqrt{N}}>\frac{2 c}{\sqrt{N}} .
$$

The lemma follows by comparing the two bounds on $\operatorname{lct}_{P}\left(S,\left.B\right|_{S}\right)$.

To conclude the proof of the theorem, we just observe that the inequalities in Lemmas 3 and 4, combined, imply that $N-3 \sqrt{N}+1<0$, a condition that is never satisfied if $N \geq 7$.

We close this note with some comments on the errors in Lemmas 9.1 and 9.2 of the original article.

Remark 1 Lemma 9.1 already fails in the following simple situation. Let $\sigma: \mathbb{A}^{2} \rightarrow \mathbb{A}^{1}$ be the projection given by $\sigma(x, y)=x$, let $P \in \mathbb{A}^{2}$ be the origin in the coordinates $(x, y)$, and let $P^{\prime}=\sigma(P)$. Let $X=\left(y+x^{2}+y^{2}=0\right) \subset \mathbb{A}^{2}$, and consider the divisor $E=[P]$ on $X$. Note that $\mu=\operatorname{val}_{E}(P)=1$ and $W=W^{1}(E)$ is the fiber of $J_{\infty} X \rightarrow X$ over $P$. Moreover, $\left.\operatorname{val}_{E}\right|_{\mathbb{C}\left(\mathbb{A}^{1}\right)}=\operatorname{val}_{E^{\prime}}$ where $E^{\prime}=\left[P^{\prime}\right]$, and $W^{\prime}=W^{1}\left(E^{\prime}\right)$ is the fiber of $J_{\infty} \mathbb{A}^{1} \rightarrow \mathbb{A}^{1}$ over $P^{\prime}$. In particular,

$$
\left(\left(W^{\prime}\right)^{0}\right)_{1}=\left(W^{\prime}\right)_{1}=T_{P^{\prime}} \mathbb{A}^{1} .
$$


In the coordinates $\left(x, y, x^{\prime}, y^{\prime}, x^{\prime \prime}, y^{\prime \prime}, \ldots\right)$ of $J_{\infty} \mathbb{A}^{2}$, the ideal of $W$ contains the elements $x, y, y^{\prime}, y^{\prime \prime}+2\left(x^{\prime}\right)^{2}$. Fix any integer $m \geq 2$. Since the element $y^{\prime \prime}+2\left(x^{\prime}\right)^{2}$ is in the ideal of $W_{m}$, after taking the degeneration to homogeneous ideals as in the proof of Lemma 9.1, the ideal of $\left(W_{m}\right)^{0}$ contains the elements $x, y, y^{\prime},\left(x^{\prime}\right)^{2}$. Therefore $\left(\left(W_{m}\right)^{0}\right)_{1}=\{0\} \subset T_{P} \mathbb{A}^{2}$ (set-theoretically), and hence

$$
\sigma_{1}\left(\left(\left(W_{m}\right)^{0}\right)_{1}\right)=\{0\} \in T_{P^{\prime}} \mathbb{A}^{1} .
$$

This shows that the lemma does not hold in this case.

The error in the proof of Lemma 9.1 is in the last formula. The formula is true before taking closures (namely, we have $\pi_{m, 0}^{-1}(P) \cap T_{P}^{(m)} \mathbb{A}^{n}=T_{P}^{(m)} X$ ), but after taking closures in the projective space we only get an inclusion $\overline{\pi_{m, 0}^{-1}(P)} \cap$ $\overline{T_{P}^{(m)} \mathbb{A}^{n}} \supset \overline{T_{P}^{(m)} X}$. In the example discussed above, for instance, the point $(0: 0: 0: 0: 1)$ in the homogeneous coordinates $\left(u: x^{\prime}: y^{\prime}: x^{\prime \prime}: y^{\prime \prime}\right)$ belongs to the closure of $W_{2}$ (which is the same as $\pi_{2,0}^{-1}(P)$ ), but not to the closure of $T_{P}^{(2)} X$.

Remark 2 The error in the proof of Lemma 9.2 is in the wrong assertion that the image under a finite morphism of a Cohen-Macaulay scheme is Cohen-Macaulay. This fails for instance for general projections to $\mathbb{P}^{4}$ of most projective surfaces in $\mathbb{P}^{5}$.

\section{References}

1. de Fernex, T., Ein, L., Mustaţă, M.: Bounds for log canonical thresholds with applications to birational rigidity. Math. Res. Lett. 10(2-3), 219-236 (2003)

2. de Fernex, T., Ein, L., Mustaţă, M.: Multiplicities and log canonical threshold. J. Algebraic Geom. 13(3), 603-615 (2004) 